EPJ Web of Conferences 60, 20018 (2013)

DOI: $10.1051 /$ epjconf $/ 20136020018$

(C) Owned by the authors, published by EDP Sciences, 2013

\title{
On equivalence of gluon-loop exchange in the inelastic processes in pertur- bative QCD to pion exchange in $\phi^{3}$ theory
}

\author{
Igor Sharf ${ }^{1, a}$, Andrii Tykhonov, b, Maksym Delyiergyiev², Grygorii Sokhrannyi ${ }^{1}$, Natalia Podolyan ${ }^{1}$, Kyrylo \\ Merkotan $^{1}$, D. Ptashynskyy ${ }^{1}$, Y. Volkotrub ${ }^{1}$, Oleksiy Potiyenko ${ }^{1}$, et Vitaliy Rusov ${ }^{1,3, c}$ \\ ${ }^{1}$ Odessa National Polytechnic University, Shevchenko av. 1, 65044 Odessa, Ukraine \\ ${ }^{2}$ Jozef Stefan Institute, Jamova 39, SI-1000 Ljubljana, Slovenia \\ ${ }^{3}$ Bielefeld University, Universitatsstrasse 25, 33615 Bielefeld, Germany
}

\begin{abstract}
We consider the hadron-hadron inelastic scattering in the framework of QCD perturbation theory. It is shown that in QCD, due to conservation of color, the tree-level diagrams of inelastic scattering are prohibited and one has to deal with the diagrams with loops. We examine the simplest type of such diagrams, where the diagram can be split into blocks, so that the integration over four-momenta of virtual particles in each block can be done independently. It is shown that for these diagrams the squared absolute value of scattering amplitude has a maximum point, similar to that observed earlier in $\phi^{3}$ model, if one takes into account the relations between the arguments of scattering amplitude, imposed by the energy-momentum conservation law. This enables to apply the Laplace's method for the calculation of cross section of hadron-hadron inelastic scattering. It is shown that the diagrams of gluon-loop exchange in QCD are equivalent to the diagrams of pion exchange in $\phi^{3}$ theory, whereby the new mechanism of cross section growth, discovered earlier in $\phi^{3}$ theory, takes place also in the perturbative QCD. The latter may explain the origin of experimentally-observed growth of cross section of hadron-hadron inelastic scattering as function of energy of colliding hadrons. The discovered mechanism can't emerge in any Regge-based model due to the premises on the particle kinematics, made in these models.
\end{abstract}

\section{Introduction}

In Ref. [1] the applicability of the Laplace's method to the calculation of the inelastic scattering cross section was demonstrated on the example of Feynman diagrams of effective scalar field theories. The aim of this work is to apply this method to a more realistic theory of hadron interactions, i.e. to quantum chromodynamics (QCD). At this point, the problem occurs due to the fact that in QCD perturbation theory one deals with the diagrams of interacting quarks and gluons, while hadrons in the initial and the final states represent the bound states of quarks and gluons. This problem is usually overcome by representing the state of interacting hadrons via the parton distribution functions (see Ref. [2] for review), which is the result of bringing into theory a large number of adjustable parameters. However, it is well known that the static hadrons can be described within the non-relativistic quark models, in which hadrons are treated as those that consist of a given number of quarks (see f.ex. Ref [3]). In these models, interaction of quarks is described by means of potential energy, ignoring the existence of gluon field inside hadrons.

Next, in the formulation of scattering problem, particles in the initial or the final states do not interact with

a. e-mail: sharph@ukr.net

b. e-mail: andrii.tykhonov@ijs.si

c. e-mail: siiis@te.net.ua each other. However, one can not neglect the interaction of quarks inside initial and final state hadrons, since this interaction keeps them bound. In the perturbation theory, one usually considers the scattering problem in the interaction picture. In this picture, the non-relativistic interaction operator is described as an integral of oscillating function, which, according to the Riemann-Lebesgue theorem, approaches zero when time approaches either plus or minus infinity, which corresponds to the initial and the final states, respectively. Hence, one can treat initial and final state quarks to be free, though they are bound. In the nonrelativistic approximation hadron's mass must be equal to sum of masses of component quarks. Neglecting all interactions with respect to the strong interaction, these masses can be considered approximately equal, i.e. we assume the hadron mass is shared equally among the constituent quarks. That is, for example, quarks in baryons are considered to have an "effective" mass which is equal to one third of the baryon's mass in the rest frame. It is found that in the hadron's rest frame its energy momentum four-vector is shared equally among quarks, but then, turning to an arbitrary frame, we get that initial and final state hadrons consist of effectively-free quarks, each of which carries either one third (baryons) or one half (mesons) of hadron's energy momentum four-vector. In this work, we consider a model in which initial and final state hadrons consist of effectively-free quarks. 


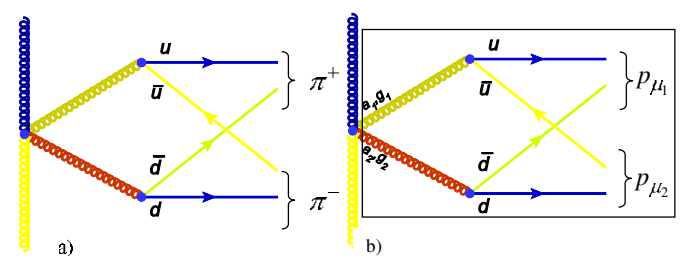

Figure 1. Elementary diagram of formation of charged pions in four-gluon interaction (left). The region that corresponds to emergence of pions is shown with the black rectangle on the diagram (right). $a_{1}, a_{2}$ and $g_{1}, g_{2}$ denote the Lorentz indices of gluons and internal indices, respectively. The four-momenta of pions are denoted with $p_{\mu_{1}}$ and $p_{\mu_{2}}$.

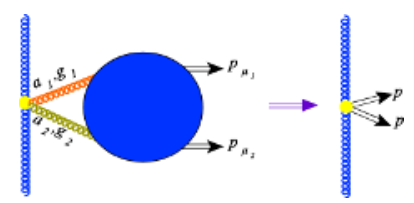

Figure 2. Generic diagram of the charged meson formation (left) and its replacement with the effective vertex (right).

The paper is structured as follows. In section 2 we demonstrate the most simple diagrams of hadron-hadron inelastic scattering, allowed in perturbative QCD. In section 3 we describe the analytical expression for scattering amplitude and address the problem of its constrained maximum. The existence of constrained maximum point is shown in this section and the most important features of scattering amplitude in the maximum point are discussed. In particular, the emergence of new mechanism of hadronhadron cross section growth is demonstrated. Conclusions are given in section 4 .

\section{The diagrams of hadron-hadron inelastic scattering}

The simplest diagram describing the formation of charged mesons in a model with effectively-free initial and final state quarks is shown in Fig. 1. To exploit the Laplace's method, one has to find the maximum point of squared absolute value of scattering amplitude. From symmetry concerns, the momenta of pions in Fig. 1 must be equal in the maximum point, i.e.

$$
p_{\mu_{1}}=p_{\mu_{2}}=p_{\mu}
$$

Furthermore, the matrix element corresponding to a diagram in Fig. 1 is a Lorentz-invariant quantity. The only Lorentz-invariant combination of $p_{\mu}$ gives $p_{\mu}^{2}$, which, due to the mass-shell condition, equals the squared pion mass $p_{\mu}^{2}=m^{2}$. The above reasoning holds also for any type of diagram, shown in Fig. 2-left. In the search for maximum point, any diagram of this type can be replaced with the effective vertex (Fig. 2-right), and the corresponding matrix element can be replaced with a constant term.

Next, due to color conservation in QCD, it is impossible to consider diagrams of inelastic scattering with a

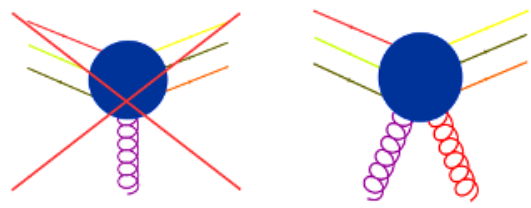

Figure 3. The "forbidden" diagram, in which the colorless system of three quark exchanges one gluon with another system (left), and the simplest diagram, allowed by the color conservation law (right).

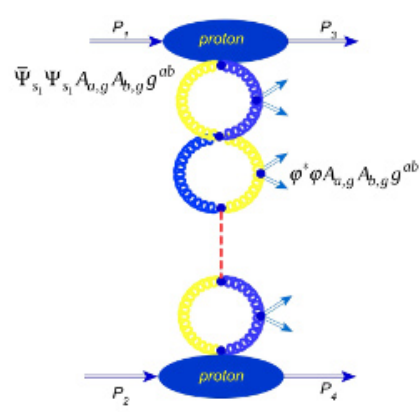

Figure 4. The simplest diagram of the proton-proton inelastic scattering involving gluon loops. The effective Lagrangians of proton-proton-gluon-gluon and pion-pion-gluon-gluon interactions are depicted on the plot.

single gluon exchange (Fig. 3-left). The simplest diagram, giving nonzero contribution to scattering amplitude, is shown in Fig. 3. In other words, the proton-proton scattering can't be described by a model with tree-level diagrams, and one has to deal with diagrams involving gluon loops. The simplest diagram of proton-proton inelastic scattering with gluon loops is shown in Fig. 4. Scattering amplitude in Fig. 4 can be represented as a product of multipliers, where each multiplier corresponds either to the proton unit or to the gluon loop. This allows to explore and to "maximize" these multipliers separately.

According to Feynman's diagram technique, scattering amplitude that corresponds to each gluon loop (Fig. 4) is

$$
A_{\text {loop }}=\int d^{4} q \frac{1}{(K-q)^{2}+i \epsilon} \frac{1}{q^{2}+i \epsilon} \frac{1}{(q-p)^{2}+i \epsilon}
$$

where $p \equiv p_{\mu_{1}}+p_{\mu_{2}}$ is the sum of momenta of final state pions in the loop, and $K$ is a momentum that enters the loop. Similar to Ref. [1], one can show that $K^{2}<0$. Applying the Feynman's identity to Eq. 2 and exploiting the negativity of $K^{2}$, the amplitude can be reduced to the following expression (see Ref. [4] for details)

$$
A_{\text {loop }}=\frac{-\pi^{2} i}{2 \sqrt{(K p)^{2}-K^{2} p^{2}}} \int d u d v \frac{1}{u v+c^{2}+i \epsilon}
$$

where

$$
c^{2} \equiv \frac{K^{2}(K-p)^{2}}{4\left((K p)^{2}-p^{2} K^{2}\right)}
$$

Eq. 3 includes an integral over the new variables $u$ and $v$. This integral can be evaluated numerically, as described 

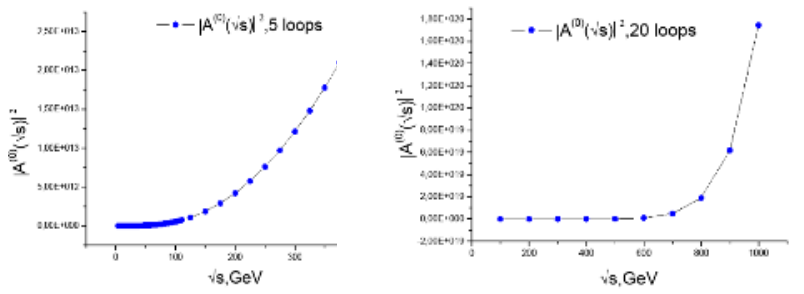

Figure 5. Squared absolute value of scattering amplitude as a function of energy of colliding protons, $\sqrt{s}$, where scattering amplitude corresponds to a diagram in Fig. 4, either with 5 loops (right) or with 20 loops (left).
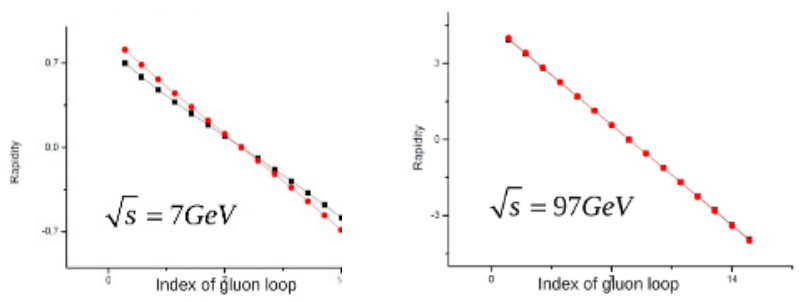

Figure 6. Rapidities of final-state particles at the maximum point as a function of position of gluon loop in the diagram (Fig. 4) : $\sqrt{s}=7 \mathrm{GeV}$ (left), $\sqrt{s}=97 \mathrm{GeV}$ (right). The rapidities form an arithmetic progression. The results of truth maximization are shown with black dots, and red dots represent the result of maximization of product of $1 / \sqrt{(K p)^{2}-K^{2} p^{2}}$ factors only.

in Ref. [4], and is insufficient to the maximization procedure. As shown below, the term $\sqrt{(K p)^{2}-K^{2} p^{2}}$ plays a significant role in behaviour of scattering amplitude at the maximum point.

\section{Exploring the maximum point of scattering amplitude}

Similar to Ref [1], from symmetry concerns, a transverse momenta of final-state particles must be zero in the maximum point. Hence, conservation of transverse momentum is automatically fulfilled and one has to ensure only that the energy and the longitudinal momentum are preserved. Longitudinal components of $P_{3}$ and $P_{4}$ (Fig. 4) can be found from these conservation laws. In order to look for the maximum point of scattering amplitude, corresponding to the diagram with $n$ loops (Fig. 4), one can treat scattering amplitude as function of $n$ variables, which are longitudinal momenta of pions in the loops. Next, it is convenient to deal with rapidities $y_{i}$ instead of longitudinal momenta $p_{i \|}$, where the rapidities are defined as $p_{i \|}=2 m \operatorname{sh}\left(y_{i}\right)$ ( $m$ denotes the pion mass). Now, one can numerically maximize a scattering amplitude. As shown in Ref. [4], scattering amplitude indeed has a maximum point upon the requirement of energy momentum conservation. The most important features of this maximum are described further in the text.

The results of numerical maximization of squared absolute value of scattering amplitude [4], which corresponds to a diagram in Fig. 4, appear to be very similar to those of the simple "comb" Feynman diagrams in the scalar $\phi^{3}$ model [1]. Furthermore, as seen from Fig. 5, the absolute value of scattering amplitude at the maximum point increases with the growth of energy of colliding hadrons. That is, the new mechanism of cross section growth, discovered earlier in the $\phi^{3}$ model [1], also takes place in the model with gluons loops. The same analogy holds also for the rapidities at the maximum point. Namely, they produce an arithmetic progression (Fig. 6), as is the case in $\phi^{3}$ theory. The similarity of this result to that of a comb diagrams in $\phi^{3}$ theory is explained as follows. As seen from Fig. 6, the results of maximization of scattering amplitude differs only slightly from those, that would have been obtained if the integrals, (Eq. 3) corresponding to each loop in diagram Fig 4, were dropped. In other words, instead of full scattering amplitude, one can deal with the product of $1 / \sqrt{(K p)^{2}-K^{2} p^{2}}$ factors only, dropping out the multipliers and constant terms, irrelevant to the maximization procedure. Furthermore, as shown in [4], at the maximum point the following approximation is relevant

$$
K_{i}^{2} \approx\left(K_{i}-p_{i}\right)^{2}
$$

Taking into account a mass-shell conditions we get the following approximation for scattering amplitude at the maximum point

$$
A \propto \prod_{i=1}^{n} \frac{1}{\sqrt{m^{2}-K_{i}^{2}}}
$$

Hence, one recovers an expression which is similar to a scattering amplitude that corresponds to a comb diagram in $\phi^{3}$ theory [4].

\section{Conclusions}

It is shown that scattering amplitude, corresponding to elementary diagrams of hadron-hadron inelastic scattering, has a maximum point upon the requirement of energy-momentum conservation. As a result, the Laplace's method can be exploited for the evaluation of cross section of hadron-hadron inelastic scattering. Next, it is shown that in QCD, scattering amplitude at the maximum point resembles exactly the same features as the one, that corresponds to the comb diagrams in $\phi^{3}$ theory. In particular, the new mechanism of cross section growth is recovered in QCD. Finally, the above mentioned similarity of QCD and $\phi^{3}$ theory is explained explicitly by the fact that the diagrams of gluon-loop exchange in QCD are equivalent to the diagrams of single-massive-particle exchange in the scalar $\phi^{3}$ theory.

\section{References}

[1] I. Sharf, A. Tykhonov, G. Sokhrannyi, M. Deliyergiyev, N. Podolyan et al., Central Eur.J.Phys. 10, 858 (2012), arXiv: 1110.4945

[2] R. Placakyte (2011), arXiv: 1111.5452

[3] L.I. Schiff, Phys. Rev. Lett. 17, 612 (1966)

[4] I. Sharf, K. Merkotan, N. Podolyan, D. Ptashynskyy, A. Tykhonov et al. (2012), arXiv: 1210.3490 